This document is the accepted manuscript version of the following article:

Quinsaat, J. E. Q., Alexandru, M., Nüesch, F. A., Hofmann, H., Borgschulte, A., \& Opris, D. M. (2015). Highly

stretchable dielectric elastomer composites containing high volume fractions of silver nanoparticles. Journal of

Materials Chemistry, 3(28), 14675-14685. http://doi.org/10.1039/C5TA03122B

\title{
Highly stretchable dielectric elastomer composites containing high volume fraction of silver nanoparticles
}

\author{
Jose Enrico Q. Quinsaat, ${ }^{a b}$ Mihaela Alexandru, ${ }^{a}$ Frank A. Nüesch, ${ }^{a b}$ Heinrich \\ Hofmann, ${ }^{b}$ Andreas Borgschulte ${ }^{c}$ and Dorina M. Opris $*^{a}$
}

The dielectric permittivity $\left(\varepsilon^{\prime}\right)$ of a polymeric material can be significantly increased when blended with conductive fillers at concentrations approaching percolation threshold. However, reproducible synthesis of such composites is after decades of research still a major challenge and a bottleneck for their application. Difficulties arise in controlling size and shape of the filler as well as in its homogenous distribution within the composite. These parameters strongly affect the dielectric as well as the mechanical properties of the composite. While a substantial amount of literature is dealing with the influence of conductive filler on the dielectric properties of composites, little is known about their mechanical properties. It is therefore still an important goal to synthesize materials with simultaneously high $\varepsilon^{\prime}$ and good mechanical properties. Here, we report the synthesis of dielectric elastomers that combine key properties such as high flexibility and stretchability, high thermal stability, increased $\varepsilon^{\prime}$, low dielectric loss and conductivity. Such materials were prepared by solution processing using quasi-spherical silver nanoparticles (AgNPs) of defined size in a polydimethylsiloxane matrix $\left(M_{w}=692 \mathrm{kDa}\right)$. To prevent percolation, the AgNPs were coated with a thin silica shell $(<4 \mathrm{~nm})$. To increase their compatibility with the silicone matrix, these core/shell nanoparticles were passivated with a silane reagent. The insulating silica shell around the particles precisely defines the minimum approach distance between the cores as twice the shell thickness. The dielectric properties of those passivated particles (filler) were measured in pellets and found to have an almost frequency independent value of $\varepsilon^{\prime}=90$ and a very low loss factor $\tan \delta=0.023$ at high frequencies. When such particles were used as filler in a polydimethylsiloxane matrix, composites with low dielectric losses were obtained. A composite containing $31 \mathrm{vol} \%$ filler with $\varepsilon^{\prime}=21$ and a $\tan \delta=$ 0.03 at $\sim 1 \mathrm{kHz}$ was achieved. At a AgNPs volume fraction of $20 \%$, the composite has a $\varepsilon^{\prime}=5.9$ at $\sim 1 \mathrm{kHz}$, a dielectric strength of $13.4 \mathrm{~V} / \mu \mathrm{m}$, elastic modulus as low as $350 \mathrm{kPa}$ at $100 \%$ strain, and a strain at break of $800 \%$. Due to the high specific energy density per volume at low electric fields, these composites are attractive materials in applications involving low electric fields.

\section{Introduction}

Next generation electronic devices require materials that combine elasticity with electronic, optoelectronic and thermal properties and open exciting challenges for material scientists. ${ }^{1}$ During the last couple of years, much research has been devoted to prepare elastomers that show increased permittivity $\left(\varepsilon^{\prime}\right)$, low dielectric losses $\left(\varepsilon^{\prime \prime}\right)$, high dielectric strength $\left(E_{b}\right)$ and high flexibility. ${ }^{2,3}$ Such elastomers are attractive dielectrics in dielectric elastomer transducers (DET) which are elastic capacitors that either elongate under a high electric field (actuator more of operation) or harvest energy upon stretching and charging (generator mode of operation). ${ }^{45}$ The amount of energy harvested per cycle increases when materials with high strain at break, high $\varepsilon^{\prime}$, and high breakdown field are used. ${ }^{6}$ However, for certain application, in the vicinity of human body, high voltages should be avoided. High $\varepsilon^{\prime}$ materials are also attractive dielectric in capacitors.

Cross-linked polydimethylsiloxanes elastomers (silicones) have excellent environmental properties, low glass transition temperature, and mechanical properties that do not change much with temperature and frequency and were intensively used by the DET community. ${ }^{7-11}$ However, the $\varepsilon^{\prime}$ of silicones is lower than 3 . Several approaches have been used to increase the $\varepsilon^{\prime}$ of a silicone by, for example, chemical modification with organic dipoles ${ }^{12-14}$ or blending with conductive fillers ${ }^{15}$ as well as high $\varepsilon^{\prime}$ ceramics. $^{16-19}$ Chemical modification of silicones with organic dipoles is a promising approach to increase $\varepsilon^{\prime}$ but other materials parameters such as flexibility, elasticity, elastic moduli, and strain at break, are also affected. Additionally, tedious synthetic steps have to be followed. Blends with ceramic fillers show also an increase in $\varepsilon^{\prime}$, however, a significant increase in $\varepsilon^{\prime}$ is obtained at volume fractions exceeding $50 \mathrm{vol} \%$ which, in turn, has a detrimental effect on elastic properties. Conductive filler composites look like a promising approach to high $\varepsilon^{\prime}$ materials since the $\varepsilon^{\prime}$ increases according to a power law as the filler concentration approaches a critical value corresponding to the percolation threshold (Equation 1): ${ }^{20}$ 


$$
\varepsilon_{r}^{\prime}=\frac{\varepsilon_{m r}^{\prime}}{\left(f_{c}-f\right)^{q}}
$$

whereby $\varepsilon_{r}^{\prime}$ and $\varepsilon_{m r}^{\prime}$ are the permittivity of the composite and of the polymer matrix respectively, $f_{c}$ is the percolation threshold fraction, $f$ is the volume fraction of the filler, and $q$ is a scaling constant. Metal particles, metal oxide particles, carbon black, carbon nanotubes, and conductive polymers have been used as conductive fillers. ${ }^{6,21-26}$

Conductive polymer fillers have the advantage of being lightweight and easy to prepare. However, when blended in a polymer matrix, the morphology of the filler is rather difficult to control and phase separation is often observed. ${ }^{27,28}$ Since permittivity is sensitive to the morphology, shape and size of the conductive filler, special care has be taken to control these parameters. ${ }^{29}$ In addition, the interfacial interaction shifts the percolation threshold toward lower volume fraction of nanoparticles and starts to be effective when the proportion between the radius of the nanoparticles and the thickness of the shell approaches a value of $10 .^{30}$ If the filler has a large size or is agglomerated, and if its size is close to that of the film thickness, discharge between electrodes happens. ${ }^{31}$ In many cases, the addition of conductive nanoparticles enhances $\varepsilon^{\prime}$ but increases the dielectric losses, making the potential gain in $\varepsilon^{\prime}$ useless. Therefore, to synthesize composites with reliable and reproducible properties, the size of the filler and its dispersion in the composites must be carefully controlled. ${ }^{32}$

Metal nanoparticles (MNPs) are easy to polarize and their shape and size can be precisely controlled and are therefore very good model systems. When applied as fillers in the above context, agglomeration of MNPs has to be avoided when dispersed into a matrix. Elsewise conductive paths can form which are detrimental to the properties. MNPs can be incorporated into a polymer matrix using in-situ or ex-situ approaches. ${ }^{33,34}$ In the former, the particles are formed through reduction or decomposition of a metal salt that was dispersed in polymer matrix. ${ }^{35}$ However, the morphology, the shape, and the size of the MNPs in the matrix is difficult to control and composites with rather low volume fraction of metal are formed. ${ }^{36,37}$ Additionally, the residual components formed during reduction/decomposition of the metal source are either left in the composite or have to be removed by tedious swelling/extraction steps. We considered this approach therefore unattractive for our goal. In the latter approach, preformed particles are blended into a polymer matrix. Here, the incompatibility and the agglomeration of the metal filler in the polymer matrix are problems that have to be overcome. Although there has been substantial amount of work devoted to the dielectric properties of MNPs polymer composites, fillers were used that were either polydisperse, or had large particles size and non-uniform shapes, as well as undefined/or no insulating coating. As mentioned above, such MNPs were not attractive for our purpose.

Silver (Ag) has a high reduction potential and can be prepared in different size and shapes and was therefore selected for our investigations. ${ }^{38,39}$ Polymer composites containing silver nanoparticles (AgNPs) are known. Shen and coworkers used AgNPs coated with an organic shell as filler in an epoxy matrix. ${ }^{40}$ The organic shells were tuned from 4-6 nm up to 8-10 nm. They showed that nanocomposites with the particles surrounded by thinner shells featured a higher $\varepsilon^{\prime}$ than the nanocomposite with thicker shells. Unfortunately, no precise description of the synthesis of such composites is given. The rather low dielectric breakdown of the composites of less than $1 \mathrm{~V} / \mu \mathrm{m}$ which is significantly lower as compared to the epoxy matrix used $(30 \mathrm{~V} / \mu \mathrm{m})$, clearly shows the importance of the type and the thickness of the insulating shell used. Very important is also the influence of the volume content of MNPs on the mechanical properties of the resulting materials. Unfortunately, to the best of our knowledge, no detailed characterization of the mechanical properties of composites containing high vol\% of metal filler exist. Furthermore, elastic composites containing high vol\% of MNPs that show large reversible deformations are not known.

It was thus a prime goal of the present work to provide robust access to metal particles that are not susceptible to chemical oxidation and which have defined size. The present work also aims to prepare surface-treated core/shell Ag/silica nanoparticles which we refer to as $\mathrm{Ag} @ \mathrm{SiO}_{2}$ and to use them as filler in polydimethylsiloxane (PDMS) matrix to create high-profile composite materials that allowed us investigating the influence of the filler on the mechanical and dielectric properties of the composite. This would allow us elucidating how much the $\varepsilon^{\prime}$ can be increased and how other properties like dielectric loss, conductivity and dielectric breakdown are affected, keeping an eye also on the mechanical properties. The preparation of large amounts of AgNPs was possible by using the polyol synthesis, while the silica coating was achieved by using an optimized Ströber method. ${ }^{41,42}$ To ensure optimum performance of the composite, the compatibility of the $\mathrm{Ag} @ \mathrm{SiO}_{2}$ particles with the PDMS matrix was significantly increased by treating the surface of the particles with hexamethyldisilazane (HMDS). Composites of surface-treated $\mathrm{Ag} @ \mathrm{SiO}_{2}$ filler in PDMS were made by solution processing. The dielectric and mechanical properties of different vol\% of $\mathrm{Ag} @ \mathrm{SiO}_{2}$ filler in PDMS were investigated for the first time.

\section{Experimental Section}

\section{Materials}

$\mathrm{AgNO}_{3}$, poly(vinyl pyrrolidinone) (PVP), ethylene glycol (EG), octamethylcyclotetrasiloxane $\quad\left(\mathrm{D}_{4}\right), \quad \mathrm{H}_{2} \mathrm{SO}_{4}, \quad$ hexamethyl disilazane (HMDS), ammonia $\left(\mathrm{NH}_{4} \mathrm{OH}\right)$, and dibutyltindilaurate $(\mathrm{Sn})$ catalysts were purchased from Aldrich and used as received. Linear hydroxyl end-functionalized polydimethylsiloxane (PDMS) $\left(M_{w}=139 \mathrm{kDa}\right)$ by ABCR (25$35 \% \quad$ mehylhydrosiloxane)-dimethylsiloxane-copolymer (AB109380) cross-linker by ABCR. Higher molecular weight hydroxyl end-functionalized polydimethylsiloxane $\left(M_{w}=692\right.$ $\mathrm{kDa})$ was prepared according to the literature. ${ }^{43}$

\section{Methods}

The nanoparticles were observed by SEM on a FEI NovaNanoSEM 230 using a BSED detector, TEM analysis were done with Philips CM30 TEM and JEOL 2200FS TEM/STEM, UV-vis absorption spectra were recorded with a 
Cary 50 spectrophotometer, DLS were done with a Malvern Zetasized Nano ZS, the thermogravimetric analysis (TGA) was conducted with a Perkin Elmer TGA7 at a heating rate of $20^{\circ} \mathrm{C}$ $\mathrm{min}^{-1}$ under a helium gas flow. In-situ XPS surface analysis are performed in a modified VG EscaLab spectrometer with the base pressure $<1 \times 10^{-10}$ mbar. XPS spectra were collected with a SPECS PHOIBOS 100 analyzer using a non-monochromated X-ray source (Al $\mathrm{K} \alpha$ : $1486.6 \mathrm{eV}$, twin anode $\mathrm{Mg} \mathrm{K} \alpha / \mathrm{Al} \mathrm{K} \alpha$ ). Image analysis was used for the TEM micrographs to estimate the particles size. The number of particles measured was about 400 for Ag core and about 100 for the coated particles with the help of a ruler for Windows. For the determination of the amount of silver in the composites, about $50 \mathrm{mg}$ of material was transferred to a pre-cleaned quartz vessel. Thereafter, $3 \mathrm{~mL}$ of nitric acid $(65 \%)$ and $1 \mathrm{~mL}$ of $\mathrm{H}_{2} \mathrm{O}_{2}(30 \%)$ was added to the components. The contents were transferred into a microwave vessel which contained $5 \mathrm{~mL}$ of deionized water (Milli-QQuality) and $1 \mathrm{~mL} \mathrm{H}_{2} \mathrm{O}_{2}$. The nanocomposite was decomposed in the microwave MLS 1200. The clear solution was transferred into a $15 \mathrm{~mL}$ PP Centrifuge tube with deionized water and diluted to $10 \mathrm{~mL}$. For the quantification of the silver content in the nanocomposite, the prepared solution was once again diluted by a factor of 100 . Standard silver solutions containing $1,2,5,10,20$ and $50 \mathrm{mg} / \mathrm{L}$ prepared from a commercially available $1 \mathrm{~g} / \mathrm{L}$ silver standard solution were used for the calibration of the ICP-OES (Vista pro Varian). The particle dispersions in toluene were prepared with a tip sonicator (Sonics VCX-500 Model CV33).

The microstructures of the films were measured with SEM on samples prepared by the freeze-broken in liquid nitrogen. Permittivity measurements were done in the frequency range of $1 \mathrm{~Hz}$ to $1 \mathrm{MHz}$ using an Novocontrol Alpha-A Frequency Analyzer using a Hewlett Packard 16451B dielectric test fixture equipped with round electrodes. Prior to the measurements, $\mathrm{Au}$ electrodes with a thickness of $50 \mathrm{~nm}$ were sputtered on both sides of the films. The diameter of the electrodes was $12.5 \mathrm{~mm}$ (A series), $7 \mathrm{~mm}$ (B series) or $21 \mathrm{~mm}\left(\mathbf{B}_{\mathbf{2 0}}\right)$. To ensure a good contact and a uniform "pressure" over the samples (because of the round electrode used), gold plates slightly smaller than the coated electrodes were used. No dependence of the dielectric properties with the electrode size was observed, which is supportive for homogenous materials. The amplitude of the probing ac electric signal applied to the samples was $1 \mathrm{~V}$. The permittivity was determined from the capacitance: $\mathrm{C}=\varepsilon \varepsilon_{0} A / d$, where $A$ is the electrode area, $d$ is the thickness of the capacitor, and $\varepsilon_{0}$ is the vacuum permittivity.

The tensile tests were performed using a Zwick Z010 tensile test machine with a crosshead speed of $500 \mathrm{~mm} / \mathrm{min}$. Tensile test specimens with a gauge width of $2 \mathrm{~mm}$ and a gauge length of $18 \mathrm{~mm}$ were prepared by die cutting. The strain was determined using a traverse moving sensor. The curves were averaged from 3 independent experiments. The tensile modulus was determined from the slope of the stress-strain curves using a linear fit to the data points within 10\% strain. Dynamic mechanical analysis was carried out on a RSA 3 DMA from TA Instruments. Stripes of $10 \mathrm{~mm}$ wide, $24 \mathrm{~mm} \mathrm{long}$, and $58 \mu \mathrm{m}$ thick were measured under a dynamic load of $2 \mathrm{~g}$, at $1 \%$ strain in the frequency range of 0.01 to $10 \mathrm{~Hz}$, at $26{ }^{\circ} \mathrm{C}$, and $65 \%$ humidity.

\section{Synthesis of AgNPs}

In a $250 \mathrm{~mL}$ round-bottom flask, PVP $\left(M_{w}=40 \mathrm{kDa}\right)$ was dissolved in EG $(100 \mathrm{~mL})$ under sonication. After complete dissolution of the polymer, the flask was stirred in an oil bath at $130{ }^{\circ} \mathrm{C}$ for $30 \mathrm{~min}$, then an aqueous solution of $\mathrm{AgNO}_{3}(2 \mathrm{~mL}$, $1 \mathrm{~g} / \mathrm{mL}$ ) was injected rapidly and the reaction mixture was stirred further for $1 \mathrm{~h}$ at a speed of $900 \mathrm{rpm}$. The flask was later removed from the oil bath, cooled in a water bath and the reaction mixture was diluted with acetone, centrifuged at 5'000 rpm for $1 \mathrm{~h}$ and decanted. The resulting precipitate was further washed with acetone and water 3 times before redispersion in EtOH. The reaction was repeated 20 times and the products were redispersed in $1.5 \mathrm{~L}$ of EtOH. Yield: $14.2 \mathrm{~g}(57 \%)$

\section{Synthesis Ag@SiO 2 core-shell particles}

The silica-coating was performed based on existing protocols of the modified Stöber method. ${ }^{41}$ From the stock solution of AgNPs prepared as described above, $200 \mathrm{~mL}$ was filled in a $1 \mathrm{~L}$ flask and diluted to $600 \mathrm{~mL}$ with EtOH. The suspension was treated with $\mathrm{NH}_{4} \mathrm{OH}(29 \%, 25 \mathrm{~mL})$ and with an ethanolic solution of TEOS (50 mL, 0.6 vol\% TEOS) and stirred at $25^{\circ} \mathrm{C}$ for $19 \mathrm{~h}$. The mixture was diluted with acetone, centrifuged at 5'000 rpm for $30 \mathrm{~min}$, decanted, and redissolved in EtOH. The products were washed with acetone for further 3 times before redispersion in EtOH. The reaction was performed 7 times to obtain $\mathrm{Ag} @ \mathrm{SiO}_{2}$ core-shell particles with a very thin silica shell of $3.3 \pm 0.7 \mathrm{~nm}(\sim 100$ particles measured $)$.

\section{Synthesis of $\mathrm{Ag} @ \mathrm{SiO}_{2} @ \mathrm{Si}\left(\mathrm{CH}_{3}\right)_{3}$}

The ethanolic suspension of $\mathrm{Ag} @ \mathrm{SiO}_{2}$ core-shell particles was centrifuged. Most of the ethanol was removed by decantation and the residue was redispersed in anhydrous toluene $(200 \mathrm{~mL})$ and stirred at $500 \mathrm{rpm}$. To this suspension, hexamethyl disilazane (HMDS) (23 $\mathrm{mL}$ ) was added and stirred for $6 \mathrm{~h}$. The particles were recovered by centrifugation, washed once again with toluene to obtain the surfacetreated $\mathrm{Ag} @ \mathrm{SiO}_{2}$ core-shell particles. Yield: $13.5 \mathrm{~g}(>95 \%)$.

\section{Synthesis of composites of series A}

The Ag@SiO $@$ @i $\left(\mathrm{CH}_{3}\right)_{3}$, PDMS ( $\left.M_{w} \sim 139 \mathrm{kDa}\right)$, and $(25-35 \%$ mehylhydrosiloxane)-dimethylsiloxane-copolymer cross-linker were mechanically mixed to a homogenous dispersion. Toluene was used to adjust the viscosity of the mixture. The dibutyltindilaurate was then added and films were prepared by doctor blading. The samples were aged for about one month before testing. For the amounts used please see Table 2.

\section{Synthesis of composites of series B}

The Ag@SiO $2 @ \mathrm{Si}\left(\mathrm{CH}_{3}\right)_{3}$ was first dispersed in toluene by using a tip sonication operated at a power of $40 \%$ for at least $10 \mathrm{~min}$. To this, PDMS $\left(M_{w}=692 \mathrm{kDa}\right)$ and $(25-35 \%$ mehylhydrosiloxane)-dimethylsiloxane-copolymer cross-linker 
were added and a homogenous composite was made by mechanically mixing. The Sn-catalyst was then added and films were prepared by doctor blading. The samples were aged for about one month before testing.

\section{Synthesis of composite $B_{\mathbf{3 1}}$}

The Ag@SiO $@ @ S i\left(\mathrm{CH}_{3}\right)_{3}$ filler, PDMS $\left(M_{w}=692 \mathrm{kDa}\right)$, and (25-35\% mehylhydrosiloxane)-dimethylsiloxane-copolymer cross-linker were mechanically mixed to a homogenous dispersion using cyclohexane solvent to adjust the viscosity. After obtaining a good dispersion the catalyst was added. Cyclohexane was selected as solvent, because it is easily to be removed in high vacuum at room temperature and therefore the speed of cross-linking reaction is lowered. To avoid agglomeration of the particles, a sonicator bath was used during the evaporation. A sticky powder formed after the solvent evaporation which was pressed in pellets by using an IR press, heated at $80{ }^{\circ} \mathrm{C}$ for $5 \mathrm{~h}$. Platinum electrodes were sputtered on the pellet before the dielectric measurements were done.

\section{Results and discussion}

\section{Synthesis and characterization of the filler particles}

We selected Ag as metal source because AgNPs are stable against oxidation and can be prepared in different sizes and shapes. Since other metal nanoparticles such as aluminum, zinc, iron, nickel and copper form an insulating oxide layer when exposed to air, we did not consider using them because their dielectric properties might change in time or when exposed to elevated temperatures due to surface oxidation. ${ }^{44-48}$

AgNPs were prepared by using the so called polyol synthesis. Briefly, an aqueous silver nitrate solution was injected into a preheated solution of polyvinyl pyrrolidone (PVP) stabilizer in ethylene glycol (EG) at $130^{\circ} \mathrm{C}$ for $60 \mathrm{~min}$. The particles were purified by washing several times with ethanol/acetone. Figure 1 shows the TEM images of the prepared AgNPs. The average particles size was $57 \pm 10 \mathrm{~nm}$ and DLS confirmed a reasonable size distribution (Figures 2 and 3). Dried particles kept in normal atmosphere for four months did not show any change in their chemical composition as proved by XRD and XPS spectra where no signals typical for silver oxide were observed (see Supp. Inform.). The particles have a cubic structure with peaks at 20: 38.1 (111), 44.3 (200), 64.5 (220), 77.4 (311), and 81.5 (222) that are in agreement with the published data (see Supp. Inform.). ${ }^{49}$ The XPS analysis shows a peak at $368.2 \mathrm{eV}$ due to metallic silver. A small peak at $365.8 \mathrm{eV}$ due to surface silver atoms surrounded by the PVP chain and no peak at $367.4 \mathrm{eV}$ typical for silver oxide was observed. It can be concluded that the polyol synthesis, which is performed at temperatures exceeding $100^{\circ} \mathrm{C}$ under normal atmosphere, does not lead to an oxidation of the silver surface neither during the synthesis nor during storage of the particles in the laboratory.

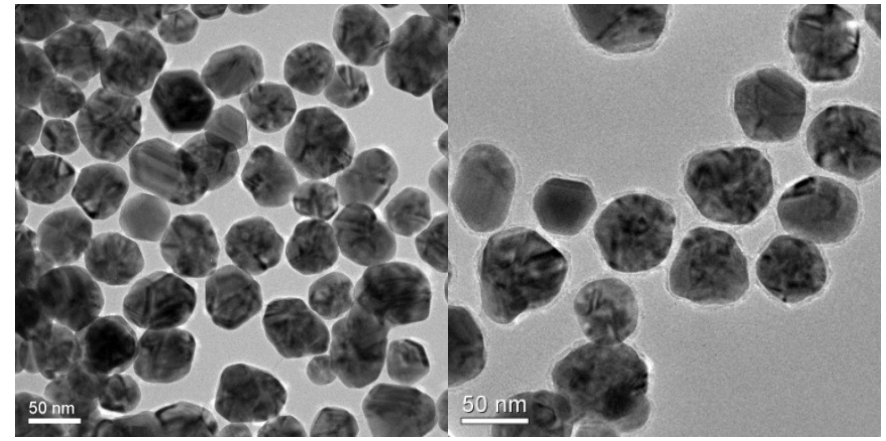

Figure 1. TEM micrographs of AgNPs prepared by reduction of $\mathrm{AgNO}_{3}$ with ethylene glycol (left) and of their coating with a thin silica shell of about $3.3 \mathrm{~nm}$ (right).

To avoid conductive paths through the material, AgNPs were surface-coated with an insulating shell prior blending. Instead of using an oxidation process, we used a hydrolysis step for the coating. We have recently showed that it is possible to synthesize AgNPs coated with a thin insulating silica shell and also to scale-up this procedure. ${ }^{50}$ Silica is an excellent insulator and has a good compatibility with the silicone matrix. A slightly modified so-called Ströber method was used. ${ }^{51}$ Our previous investigations have shown that a silica shell thickness of less than $4 \mathrm{~nm}$ is optimal to avoid electrical conductivity and to increase $\varepsilon^{\prime}$ of the resulting nanocomposites concurrently. ${ }^{50}$ This coating was achieved by dispersing the AgNPs at a certain concentration in ethanol and let tetraethoxysilane (TEOS) to hydrolyze under basic conditions and deposit on the Ag surface. The silica-coating is facilitated by the presence of residual PVP around the Ag cores since it serves as an anchoring group for the growing $\mathrm{SiO}_{2}$ layer. ${ }^{51,52}$ The successful coating was proven by TEM, UV-vis, DLS and EDX. TEM images show a strong contrast between the black silver cores and the grey silica shell and clearly proves the formation of $\mathrm{Ag} @ \mathrm{SiO}_{2}$ core/shell structures (Figure 1). Further evidence for the coating is provided by surface plasmon resonance. Upon coating the AgNPs with a thin silica shell $(3.3 \pm 0.7 \mathrm{~nm})$, a slight bathochromic shift of the absorption peak from $\lambda=436 \mathrm{~nm}$ to $\lambda$ $=437 \mathrm{~nm}$ in the UV-vis spectra is observed (Table 1 and Figure 2) as a result of the higher dielectric constant of $\mathrm{SiO}_{2}$ as the new medium compared to PVP/EtOH. DLS also confirms a slight increase in the hydrodynamic radius after the silicacoating of the particles (Figure 2). Generally, the presence of the silicon peak in the EDX of the coated AgNPs further supports the presence of the silica shell (see Supp. Inform.). It is important to note that each individual AgNPs is coated with a silica shell of about the same thickness and also that no agglomeration of the AgNPs occurred during the coating. We can also confirm that the molecular weight of the PVP used for the synthesis is important for the functionalization of the particles with the silica shell. ${ }^{51}$ For example PVP of $M_{\mathrm{w}}=40$ $\mathrm{kDa}$ allowed formation of uniform silica shells as compared to PVP of $M_{w}=10 \mathrm{kDa}$ where the silica shell was less uniform. This effect is even more pronounced when a very thin silica 
shell is desired. Thin uniform silica shells are more difficult to prepare than the thick shells. According to the literature, lower molecular weight PVP might even lead to the agglomeration of the core-shell particles due to the lack of shielding off the silver cores from the attractive van der Waals forces. ${ }^{25}$

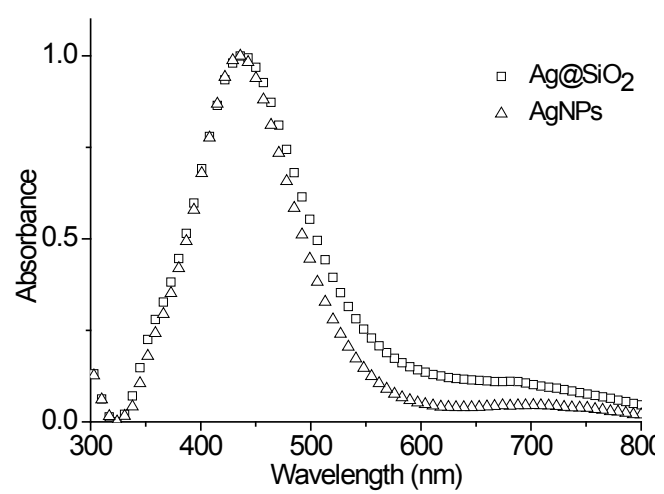

Figure 2. UV-vis spectra of $\operatorname{AgNPs}(\Delta)$ and silica-coated $\mathrm{Ag} @ \mathrm{SiO}_{2}$ core-shell particles $(\square)$ recorded in ethanol.

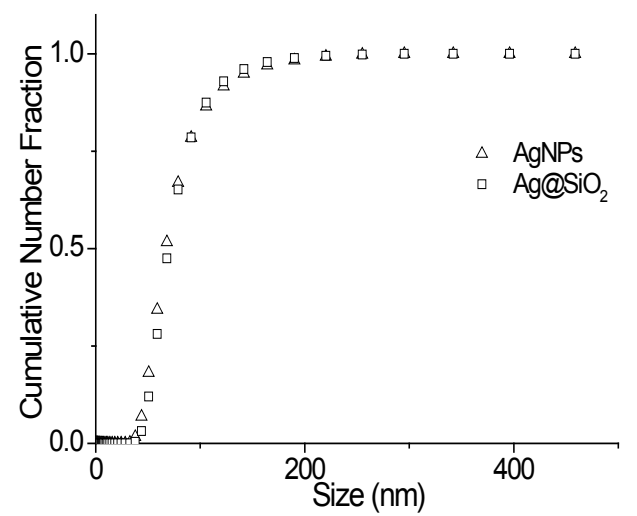

Figure 3. DLS spectra of $\operatorname{AgNPs}(\Delta)$ and silica-coated $\mathrm{Ag} @ \mathrm{SiO}_{2}$ core-shell particles $(\square)$ recorded in ethanol.

Table 1. Size and $\lambda_{\max }$ data of AgNPs and $\mathrm{Ag} @ \mathrm{SiO}_{2}$ obtained by TEM, DLS and UV-vis of the core-shell particles before and after the surface treatment with hexamethyldisilazane.

\begin{tabular}{cllll}
\hline Entry & Sample & $\begin{array}{l}\text { TEM } \\
{[\mathrm{nm}]}\end{array}$ & $\begin{array}{l}\text { DLS } \\
{[\mathrm{nm}]}\end{array}$ & $\begin{array}{l}\lambda_{\max } \\
{[\mathrm{nm}]}\end{array}$ \\
\hline 1 & $\mathrm{AgNPs}$ & $57(10)$ & $60(24)$ & 436 \\
2 & $\mathrm{Ag} @ \mathrm{SiO}_{2}$ & $64(11)$ & $68(27)$ & 437 \\
3 & $\mathrm{Ag} @ \mathrm{SiO}+\mathrm{Si}\left(\mathrm{CH}_{3}\right)_{3}$ & - & $68(26)$ & 439 \\
\hline
\end{tabular}

The silica surface is quite reactive and needs to be passivated by a hydrophobic layer prior to blending. Three different reagents were tried to achieve this: hexamethyldisilazane (HMDS), methoxy(dimethyl)octylsilane, and noctyldimethylchlorosilane. HMDS was preferred since the resulting particles $\left(\mathrm{Ag} @ \mathrm{SiO}_{2} @ \mathrm{Si}\left(\mathrm{CH}_{3}\right)_{3}\right.$ - filler) formed stable dispersions in toluene as can be seen in the wettability test (Figure 4). Furthermore, the reaction occurs seemingly fast and does not require inert conditions. ${ }^{53}$ In addition to the wettability test, the increase in the carbon content in elemental analysis of the surface-treated particles as compared to the starting one further confirms the functionalization. The elemental analysis data show an increase in the carbon content for the HMDS treated particles as compared to the silica coated one. The functionalized particles $\mathrm{Ag} @ \mathrm{SiO}_{2} @ \mathrm{Si}\left(\mathrm{CH}_{3}\right)_{3}$ were kept wet in order to avoid their agglomeration. Table 1 summarizes the sizes of the as prepared AgNPs, of $\mathrm{Ag} @ \mathrm{SiO}_{2}$, and of $\mathrm{Ag} @ \mathrm{SiO}_{2} @ \mathrm{Si}\left(\mathrm{CH}_{3}\right)_{3}$ obtained from TEM, DLS and UV-vis measurements.

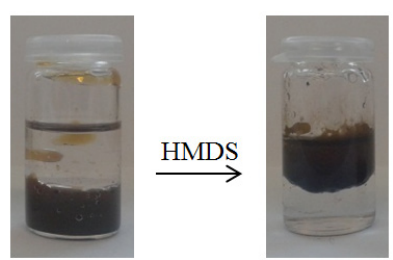

Figure 4. Wetting test of $\mathrm{Ag} @ \mathrm{SiO}_{2}$ core-shell particles conducted in toluene/water mixture before (left) and after (right) surface functionalization with HMDS. The particles are stable in time and do not sediment.

\section{Synthesis and characterization of AgNP composites}

Two series of composites were prepared and are named as $\mathbf{X y}$, where $\mathbf{X}$ represents the name of the series synthesized with PDMS of different $M_{w}$ and $\mathbf{y}$ represents the Ag vol\%. Since the particles were kept wet all the time, the amount of $\mathrm{Ag}$ in the composites was determined by Inductively Coupled Plasma-Optical Emission Spectrometry (ICP-OES) and is given as $\mathbf{y}$ in the samples' code. As matrix, a commercial and a home-made hydroxyl end-functionalized polydimethylsiloxane (PDMS) $M_{w}=139 \mathrm{kDa}$ (for Series A) and $M_{w}$ $=692 \mathrm{kDa}$ (for series B) were used, respectively. These PDMS polymers were cross-linked via a condensation reaction of the hydroxyl end-groups with the hydrosilane groups of $(25-35 \%$ methylhydrosiloxane)-dimethylsiloxane-copolymer cross-linker (CL) (see Experimental Section). Different volumes of highly concentrated filler solution in toluene were dispersed in PDMS matrix. To these mixtures the CL and the Sn-catalyst were added. Films were prepared by doctor blade technique. Depending on the $\mathrm{Ag}$ volume fraction the color of the composites changes from brown (below 10-15 vol\%) to grey (>15\%). Table 2 gives an overview of the reagents used for the synthesis of materials of series $\mathbf{A}$ and $\mathbf{B}$. The use of organotin catalyst is also known as Room Temperature Vulcanization (RTV) or 'cold vulcanization' which allows the formation of elastomers in about $24 \mathrm{~h}^{54}$ Nevertheless, the samples were aged for 1 month prior to performing the mechanical measurements to ensure the complete evaporation of residual toluene and to allow sufficient time for the finalization of the condensation reactions. Our attempts to use a silicone matrix that is cross-linked via a hydrosilylation which requires shorter reaction time at elevated temperatures were not successful.

The Scanning electron microscopy (SEM) images were recorded with a backscatter electron detector (BSED) which is designed to illustrate differences in molecular densities. Here, the particles are featured as small bright spots due to their 
higher scattering intensity compared to the PDMS matrix (Figure 5). The present particle agglomerates within the nanocomposites are depicted as large, bright ensembles. SEM images of the samples A prepared by freeze-breaking in liquid nitrogen illustrate that even at a low $\mathrm{Ag}$ vol\% some
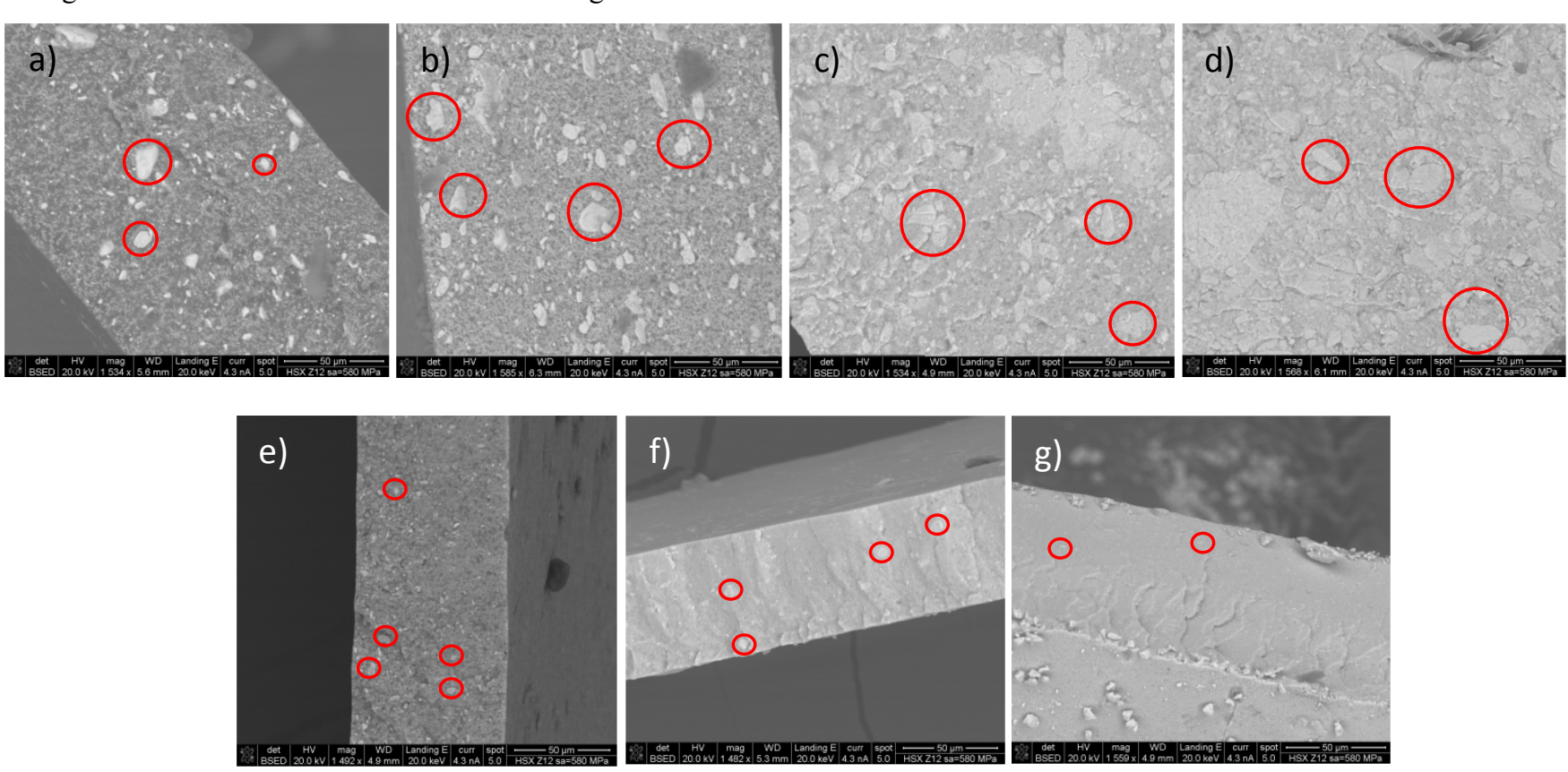

Figure 5. SEM images of the freeze-broken Ag/PDMS nanocomposites of series A containing 5, 12, 18, 25 vol\% and Ag (a-d) and of series B containing 9, 14, and 20 vol\% Ag (e-g). Some present agglomerates are shown in the red circles. For all images, the scale bar is $50 \mu \mathrm{m}$.

The mechanical properties of the composites were studied by tensile tests. The results are summarized in Table 3 . The stressstrain curves, averaged from three independent tests are shown in Figure 6. Material $\mathbf{A}_{5}$ shows an elastic behavior, while material $\mathbf{A}_{25}$ retains flexibility but it is plastically deformed. The strain at break decreases with increasing amount of Ag from about $52 \%$ for $\mathbf{A}_{5}$ to $8.8 \%$ for $\mathbf{A}_{\mathbf{2 5}}$. As expected, an increase in the stiffness of the materials with increasing the Ag vol\% was observed.

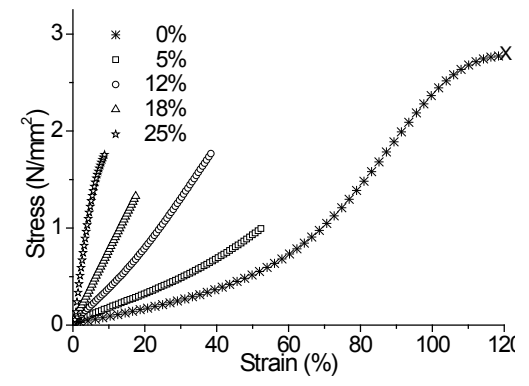

Figure 6. Stress-strain curves of the composites of series A containing different vol\% of $\mathrm{Ag}$ and of the neat matrix. The vol\% of Ag was determined after the films were cross-linked by using ICP-OES. agglomeration can be observed, while with increasing Ag vol\% more agglomerates with dimensions reaching tens of microns were observed. Films of the series $\mathbf{B}$ feature a better dispersion of the filler with fewer agglomerates. 
Toluene was used to adjust the viscosity of the composites. The amount of Ag contained in the composite was determined after cross-linking by ICP-OES. Although all composites of series A are flexible, the materials with higher than $15 \% \mathrm{Ag}$ are not elastic and could not be reversibly stretched. We therefore, next explored the possibility of preparing highly stretchable elastomers with high Ag vol\%. It is well known that the mechanical properties of an elastomer are affected by both the molecular weight of the polymer as well as the amount of cross-linker used. Therefore, to further improve the elastic properties of the composites, a hydroxyl endfunctionalized PDMS of a high molecular weight $\left(M_{\mathrm{w}}=692 \mathrm{kDa}\right.$, GPC) was used which allowed us the synthesis of composite of series B. This polymer was synthesized by cationic ring-opening polymerization of octamethylcyclotetrasiloxane $\left(\mathrm{D}_{4}\right)$ in the presence of $\mathrm{H}_{2} \mathrm{SO}_{4}{ }^{43}$ Highly stretchable and flexible materials with strain at break much higher as compared to the matrix formed (Figure 7). As expected an increase in the elastic moduli at all strains with increasing the amount of filler used was observed. The influence of the filler on the mechanical properties is more pronounced in the low strain regime, while with increasing the strain, the filler influence is less pronounced (see Figure 7 insert and Table 2). Two composites with 20 vol\% Ag but different amount of cross-linker were first prepared ( ${ }^{\text {stiff }} \mathbf{B}_{\mathbf{2 0}}$ and $\mathbf{B}_{\mathbf{2 0}}$ ) (for the amounts used, please see Table 2). Composite ${ }^{\text {stiff }} \mathbf{B}_{\mathbf{2 0}}$ has a strain at break of $270 \%$ and elastic moduli at low strains in the 6 MPs regime. For composite $\mathbf{B}_{\mathbf{2 0}}$ the amount of the cross-linker used was reduced to half. Composite $\mathbf{B}_{\mathbf{2 0}}$, despite of its high Ag vol\% content, shows excellent elastic properties with a strain at break as high as $800 \%$ and moduli of elasticity as low as $350 \mathrm{kPa}$ at $100 \%$ strain. The $\mathrm{Ag}$ vol\% content was further reduced for materials $\mathbf{B}_{\mathbf{1 4}}$ and $\mathbf{B}_{\mathbf{9}}$. These composite show a slight increase in the strain at break and decrease in the elastic moduli as compared to $\mathbf{B}_{\mathbf{2 0}}$.

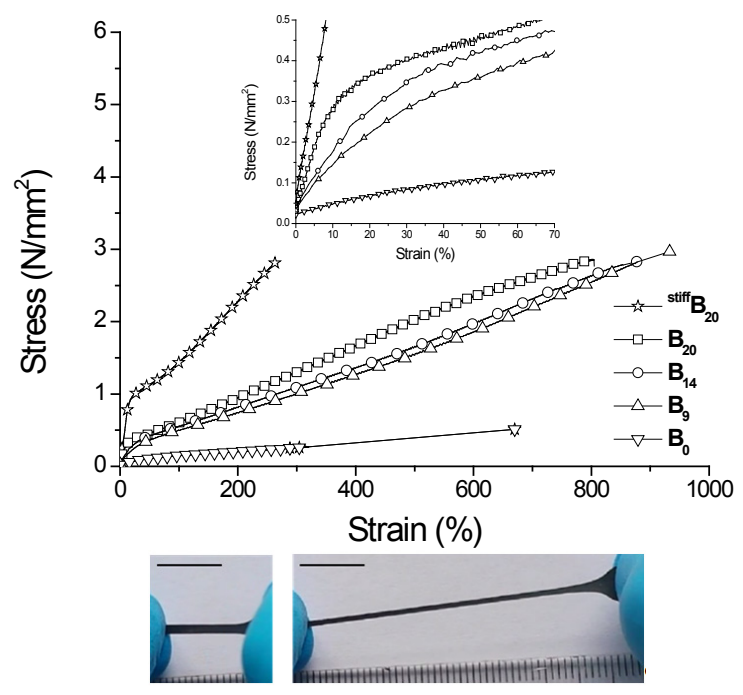

Figure 7. Stress-strain curves of the matrix $\mathbf{B}_{\mathbf{0}}$, ${ }^{\text {stiff }} \mathbf{B}_{\mathbf{2 0}}, \mathbf{B}_{\mathbf{2 0}}, \mathbf{B}_{\mathbf{1 4}}$, and $\mathbf{B}_{\mathbf{9}}$; the enlargement for the low strains (insert); and photos of $\mathbf{B}_{\mathbf{2 0}}$ in its relaxed and strained form (bottom) (the scale bar represents $1 \mathrm{~cm}$ ).
Composites $\mathbf{B}_{\mathbf{1 4}}$ and $\mathbf{B}_{\mathbf{2 0}}$ were further subjected to extensionrelaxation cycles. An initial load of $0.01 \mathrm{~N}$ was used. Figure 8 shows the behavior of a freshly prepared specimen in its first five cycles of extension-relaxation to a maximum of $50 \%$ strain. As can be seen, for the second extension-relaxation cycle the stress required on reloading is reduced as compared to the initial loading and the hysteresis observed for the subsequent cycles was rather low. The same sample was subjected to successively higher strains from $50 \%$ up to $450 \%$ followed by returning to the original gauge length (see Supp. Inform.). A strong dependence of the stress-strain curves upon increasing the strain level was observed. This stress softening phenomenon is typical for filled rubber materials and is known as the Mullins effect. Different mechanisms have been proposed to explain this effect including reorganization of the polymer network, reorganization of the filler-filler networks, and/or the detachment of the polymer network from the filler. ${ }^{55}$
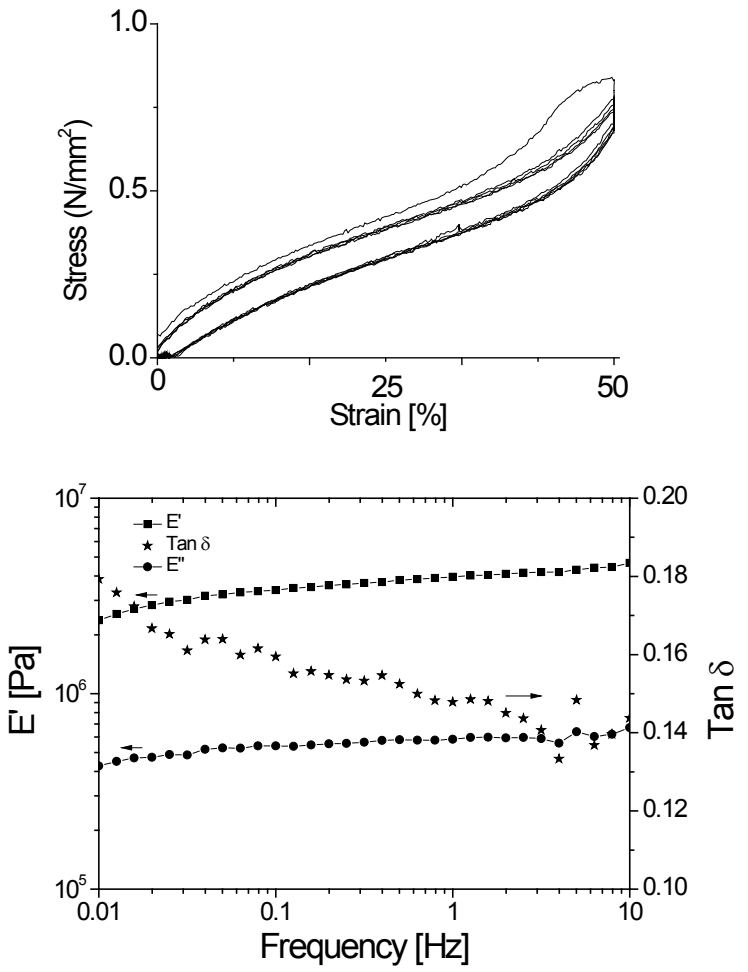

Figure 8. Cyclic stress-strain relaxation curves of $\mathbf{B}_{\mathbf{1 4}}$ at $50 \%$ strain (top) and dynamic mechanical analysis for $\mathbf{B}_{\mathbf{2 0}}$ at different frequencies (bottom).

Material $\mathbf{B}_{\mathbf{2 0}}$ was aged for 5 days at $150{ }^{\circ} \mathrm{C}$ and its stress-strain and the cyclic relaxation behavior was compared with that of a $\mathbf{B}_{\mathbf{2 0}}$ sample aged at $25{ }^{\circ} \mathrm{C}$ for 10 days (see Supp. Inform.). The elastic properties were only slightly affected by the temperature. Material $\mathbf{B}_{\mathbf{2 0}}$ is slightly stiffer after this aging and the strain at break decreased, but the elastic property is retained. The cyclic tests show some differences for the first strain-release cycle and a small difference between the cycles at the used strain. The hysteresis loop at the first strain-release cycle is more pronounced for the sample that was aged at elevated temperatures (see Supp. Inform.). 
Additionally, dynamic mechanical analysis has been carried out on $\mathbf{B}_{\mathbf{2 0}}$ (Figure 7 right). The elastic modulus measured at low strains (1\%) and at $0.01 \mathrm{~Hz}$ was $2.4 \mathrm{MPa}$ while by increasing the frequency to $0.1 \mathrm{~Hz}$ an increase in the elastic modulus to $3.4 \mathrm{MPa}$ was observed. The viscoelastic losses $(\tan \delta)$ are rather low $(\tan \delta<0.2$ at all frequencies) and further support the good elastic properties of $\mathbf{B}_{\mathbf{2 0}}$.

Silicones are generally characterized by a pronounced thermal stability and a single weight loss step at high temperatures. It has been demonstrated that PDMS degrades through the depolymerisation of PDMS to give cyclic oligomeric siloxanes. ${ }^{56,57}$ The silicone matrix used by us $\left(\mathbf{B}_{\mathbf{0}}\right)$ is stable up to $400{ }^{\circ} \mathrm{C}$ where it starts to decompose (Figure 9). The decomposition of the composites of series $\mathbf{B}$ proceeds in one step and the degradation of the polymer is shifted to values above $500{ }^{\circ} \mathrm{C}$. The incorporation of filler to the PDMS restricts the chain mobility and therefore the degradation of the nanocomposites delayed compared to the pure silicone rubber. ${ }^{58,59}$ An increase in the amount of filler does not lead to a further change in the thermal properties of the nanocomposites. As expected, the amount of residue left due to the $\mathrm{Ag} @ \mathrm{SiO}_{2}$ filler increases with increasing the $\mathrm{Ag}$ vol\% and clearly shows that the composites have high filler content. The amount of residue corresponds to the amount of $\mathrm{Ag} @ \mathrm{SiO}_{2}$ used.

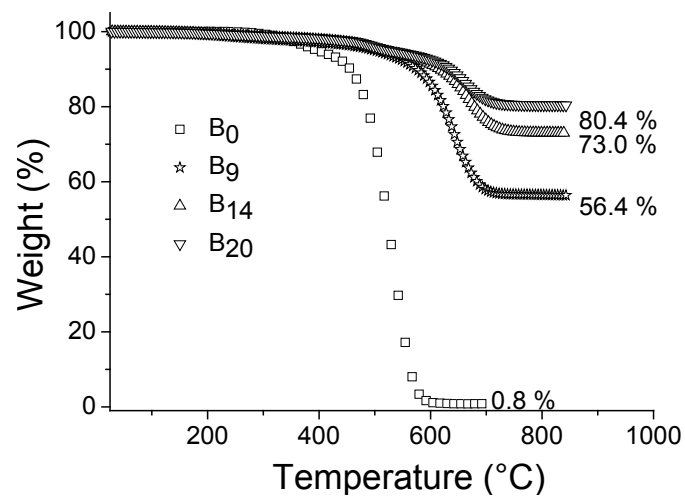

Figure 9. TGA curves of composites $\mathbf{B}$ and of the matrix under helium.

\section{Dielectric properties of the filler and composites}

The dielectric properties of the filler, matrix, and composites were measured using an impedance spectrometer in a frequency range from $1 \mathrm{~Hz}$ to $10^{6} \mathrm{~Hz}$ at $1 \mathrm{~V}$ amplitude at room temperature (Figure 10). The dielectric properties of the pure filler were measured in pellet that was obtained by pressing the dried particles using an IR press. Two platinum electrodes were sputtered on both sides. An almost constant frequency dependent $\varepsilon$ ' with a value of about 90 at $10^{3} \mathrm{~Hz}$ and rather low loss factor $\tan \delta$ at high frequencies was observed (see Table 3, Figure 10). Furthermore, the low conductivity at low frequency of $9 \times 10^{-11} \mathrm{~S} / \mathrm{cm}$ is supportive for a core/shell structure.

The composites of series $\mathbf{A}$ show an expected increase in $\varepsilon$ ' with the Ag vol\% from 3 for the silicone matrix to 4.4 for $5 \%$, to 5.6 for $12 \%$ and reaches a max value of 7.8 for $18 \%$. The dielectric losses and also the conductivity slightly increase with increasing the amount of filler, but remain at low values characteristic for dielectric materials. For example, all composites have a conductivity that is lower than $3 \times 10^{-13}$ $\mathrm{S} / \mathrm{cm}$ at low frequencies. A further increase in the AgNPs volume fraction to $25 \%$ results in inhomogeneous films with a rough surface, making difficult an accurate measurement of film thickness. ${ }^{60}$ As mentioned above, for series B, a PDMS of a higher molecular weight was used. The concentration of silver was varied between 9 and 31 vol. \% Ag. High quality films were prepared for concentrations up to $20 \%$ by the doctor blade technique. However, a further increase in the concentration of the filler resulted in non-homogenous films. To nevertheless find how the dielectric properties are changing when the filler vol\% approaches the concentration where the particles are percolating (this "mechanical" percolation should not be confused with the conductive percolation), a different strategy was used (see Experimental Section) which allowed us the synthesis of $\mathbf{B}_{\mathbf{3 1}}$. The dielectric properties of series $\mathbf{B}$ are similar to those of series A. Also here, composites with very low conductivity and low dielectric losses were obtained. Composite $\mathbf{B}_{31}$ with a $\mathrm{Ag}$ vol\% of $31 \%$ has a permittivity as high as 21 , a low loss factor of 0.03 at $1 \mathrm{kHz}$ with a value for the permittivity that remains almost constant with the frequency. A value of $\varepsilon^{\prime}=5.9$ for $\mathbf{B}_{\mathbf{2 0}}$ which is also highly stretchable. It was reported before that the conductive filler/insulator composites show a percolation like increase in the $\varepsilon^{\prime}$, however a linear increase in the permittivity with increasing the $\mathrm{Ag}$ vol\% was observed in our case (see Supp. Inform.). The increase in the $\varepsilon$ ' for the two series is slightly different. This might be due to the different ways of dispersing the filler in the polymer matrix: mechanical mixing for series $\mathbf{A}$ while tip sonication was used for series B. Through the dispersion of the filler by mechanical mixing, agglomerates in the size of tens of microns are still present within the matrix (Figure 4) while when the sonicator tip was used to disperse the particles, more homogenous dispersion of the particles within the matrix was observed. The particles within the agglomerates are in closer proximity to each other, resulting in a collective 
This document is the accepted manuscript version of the following article:

Quinsaat, J. E. Q., Alexandru, M., Nüesch, F. A., Hofmann, H., Borgschulte, A., \& Opris, D. M. (2015). Highly stretchable dielectric elastomer composites containing high volume fractions of silver nanoparticles. Journal of Materials Chemistry, 3(28), 14675-14685. http://doi.org/10.1039/C5TA03122B
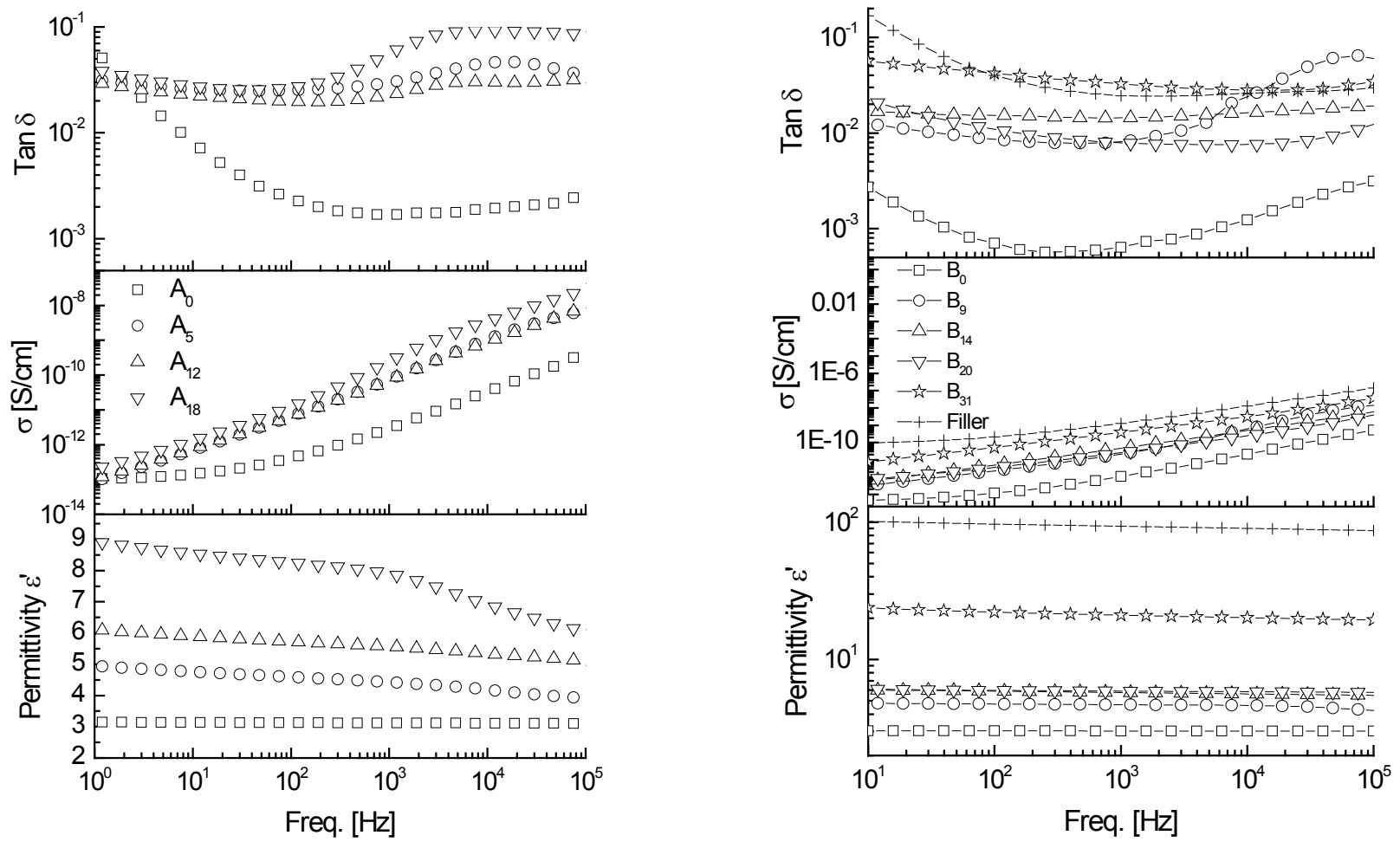

Figure 10. Permittivity, $\tan \delta$, and conductivity as function of frequency for series $\mathbf{A}$ (left) and series $\mathbf{B}$ and the filler (right).

response to the electric field from the present short-range order of the particles, which is absent in a well dispersed, agglomerate-free system. ${ }^{61}$ It has also been reported elsewhere that the presence of agglomerates within the nanocomposite leads to higher $\varepsilon$ ' values compared to the well dispersed system, as a direct result of moisture inclusion as well as specific effects resulting from the agglomerated structure. ${ }^{62}$ The breakdown field of the resulting composites was also measured (see Table 3 and Supp. Inform.). The breakdown experiments were conducted using a setup similar to that described by Kollosche $^{63}$ and also in capacitor devices that had round electrodes $(\varnothing=8 \mathrm{~mm})$. It should be also mentioned here that no actuation of the materials was observed when conducting the breakdown measurement. The dielectric breakdowns are higher for series $\mathbf{B}$ as compare to series $\mathbf{A}$ which are supportive for a better dispersion of the filler in the composites of series B as well as the increase in the molecular weight of the polymer matrix. ${ }^{60,64} \mathrm{~A}$ decrease in the breakdown field with increasing the $\mathrm{Ag}$ vol\% was observed from $23.1 \mathrm{~V} / \mu \mathrm{m}$ for $\mathbf{B}_{9}$ to 13.4 $\mathrm{V} / \mu \mathrm{m}$ for $\mathbf{B}_{\mathbf{2 0}}$.
Table 3. Summary of the densities, permittivity, loss factor, and breakdown field of the composites of series $\mathbf{A}$ and $\mathbf{B}$.

\begin{tabular}{ccccccc}
\hline Sample & ${ }^{\mathrm{a}} \boldsymbol{\varepsilon}^{\prime}$ & ${ }^{\mathrm{a}} \tan \delta$ & $\begin{array}{c}E_{b} \\
{[\mathrm{~V} / \mu \mathrm{m}]^{\mathrm{b}}}\end{array}$ & $\begin{array}{c}E_{b} \\
{[\mathrm{~V} / \mu \mathrm{m}]^{\mathrm{c}}}\end{array}$ & $\begin{array}{c}Y^{\mathrm{d}} \\
{[\mathrm{MPa}]}\end{array}$ & $\begin{array}{c}\text { Max } \\
\text { Strain } \\
{[\%]}\end{array}$ \\
\hline $\mathbf{A}_{\mathbf{0}}$ & 3.0 & 0.0017 & 77 & - & 0.78 & 119 \\
$\mathbf{A}_{\mathbf{5}}$ & 4.4 & 0.0307 & 16.9 & 11.8 & 1.43 & 52 \\
$\mathbf{A}_{\mathbf{1 2}}$ & 5.6 & 0.0234 & 8.8 & 8.8 & 3.74 & 38 \\
$\mathbf{A}_{\mathbf{1 8}}$ & 7.8 & 0.0609 & 10.4 & 3.5 & 7.51 & 17 \\
$\mathbf{B}_{\mathbf{3 1}}$ & 21 & 0.0300 & - & 1.3 & - & - \\
stiff $_{\mathbf{B}}$ & 6.8 & 0.0142 & 19.0 & 5.8 & 5.98 & 268 \\
$\mathbf{B}_{\mathbf{2 0}}$ & 5.9 & 0.0078 & 13.4 & 5.9 & 1.44 & 805 \\
\hline
\end{tabular}




\begin{tabular}{ccccccc}
\hline $\mathbf{B}_{14}$ & 5.7 & 0.0144 & 21.4 & 12.3 & 1.23 & 878 \\
$\mathbf{B}_{9}$ & 4.7 & 0.0083 & 23.1 & 29.4 & 0.89 & 935 \\
$\mathbf{B}_{\mathbf{0}}$ & 3.0 & 0.0003 & 63 & 40.0 & 0.23 & 670 \\
\hline
\end{tabular}

${ }^{\mathrm{a}}$ The values for the permittivity were taken at $\sim 1 \mathrm{kHz}$, which is sufficiently high frequency to avoid the ion conduction and interfacial polarization effects. ${ }^{\mathrm{b}}$ Measurements conducted using small electrodes $\left(1 \mathrm{~mm}^{2}\right)$. About 10 samples were measured and the data were averaged. ${ }^{\mathrm{c}}$ Measurements conducted with electrodes with a diameter of $8 \mathrm{~mm}$. "'Young's modulus calculated at a strain of $10 \%$.

The dielectric breakdown of a material is strongly affected by the elastic moduli. ${ }^{65}$ The dielectric breakdown of our composites is significantly higher as compared to the epoxy composites containing AgNPs coated with an organic shell, ${ }^{40}$ despite that herein a soft matrix was used.

As mentioned in the introduction, dielectric elastomers with increased permittivity can find applications in dielectric elastomer generator (DEG) for energy harvesting. ${ }^{66}$ For this purpose, the DEG undergoes a working cycle which involves: (a) stretching the area, (b) charging, (c) mechanical relaxation followed by (d) energy harvesting. ${ }^{6,67}$ The output of the system is quantified by the specific energy density per volume $\Delta \mathrm{W} / \mathrm{Vol}$ (Eq. 2). The specific energy density per volume of the nanocomposites for a corresponding electric field is shown in Figure 11. The parameter $\Delta \mathrm{W} / \mathrm{Vol}$ is limited by the electric breakdown field $E_{\mathrm{B}}$ of the nanocomposites and can be calculated by: ${ }^{6}$

$$
\frac{\Delta W}{V o l}=\frac{1}{2} \varepsilon \varepsilon_{0} E_{B}^{2}\left(1-\frac{1}{\left(s_{\max }+1\right)^{2}}\right)
$$

where, $\varepsilon_{0}$ is the vacuum permittivity and $s_{\max }$ is the maximum strain at break for the corresponding elastomers. As shown in Figure 11, the breakdown field of the nanocomposites is lower as compared to the silicone matrix and despite of the increase in the permittivity and the high strain at break, the maximum amount of energy that can be harvested with these composites is lower as compared to the silicone matrix. However, the amount of energy harvested at low electric fields is higher for composites $\mathbf{B}_{\mathbf{9}}$ and $\mathbf{B}_{\mathbf{1 4}}$ as compared to the matrix. For example at an electric field of $20 \mathrm{~V} / \mu \mathrm{m}$ the maximum amount of energy per cycle increases from $5.2 \mathrm{~mJ} / \mathrm{cm}^{3}$ for the $\mathbf{B}_{\mathbf{0}}$ silicone matrix, to $8.2 \mathrm{~mJ} / \mathrm{cm}^{3}$ for $\mathbf{B}_{\mathbf{9}}$ and to $10 \mathrm{~mJ} / \mathrm{cm}^{3}$ for $\mathbf{B}_{\mathbf{1 4}}$. Therefore, these nanocomposites might be useful as dielectric in energy harvester operated at low electric fields.

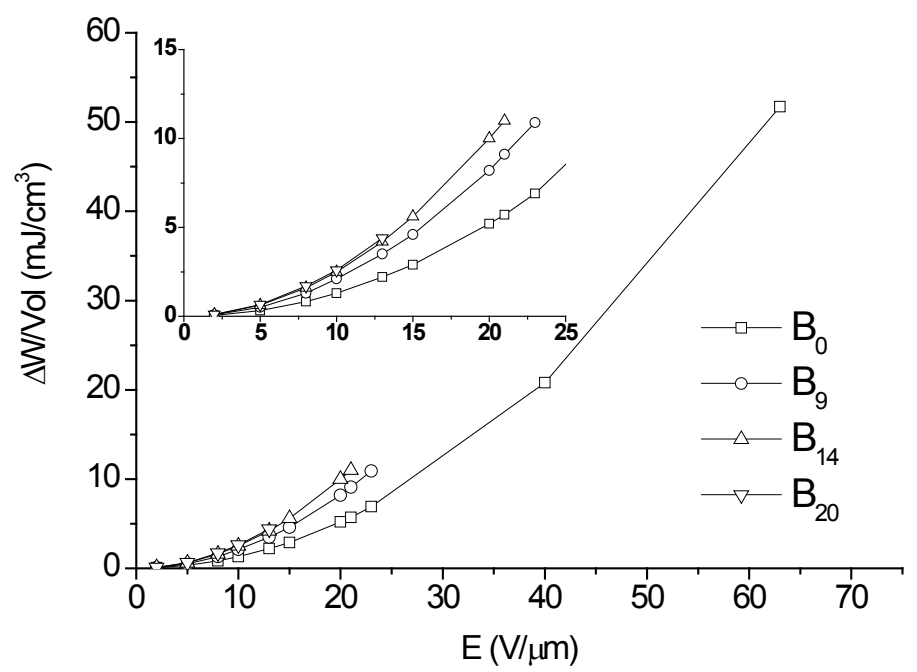

Figure 11. Specific energy density per volume at a given electric field.

\section{Conclusions}

Core/shell Ag/silica nanoparticles with Ag core of $57 \mathrm{~nm}$ surrounded by a thin silica shell of $\sim 4 \mathrm{~nm}$ and passivated by a trimethylsilyl layer show a $\varepsilon^{\prime}-=-90$ very low dielectric losses. These particles were used as filler in a polydimethylsiloxane matrix to synthesize composites with different Ag vol\%. It was shown that by a careful selection of matrix and cross-linker amount, it is possible to prepare materials with concentrations of filler as high as $20 \mathrm{vol} \%$ that have an elastic modulus as low as $350 \mathrm{kPa}$ at $100 \%$ strain and a strain at break as high as $800 \%$. The stress relaxation tests show a minor hysteresis from the first to the second cycle and almost no hysteresis for the subsequent cycles. Additionally, composites with increased $\varepsilon$ ', low dielectric losses, and high dielectric breakdowns were obtained. An increase in the $\varepsilon$ ' with increasing the vol\% of $\mathrm{Ag}$ was observed. For example, a composite containing $31 \mathrm{vol} \%$ filler has a $\varepsilon^{\prime}=21$ and a loss factor of 0.03 at $\sim 1 \mathrm{kHz}$. The low dielectric losses of these materials are supportive of a good encapsulation of the metallic Ag in an insulating silica shell. A composite containing $20 \mathrm{vol} \%$ AgNPs has a $\varepsilon^{\prime}=5.9$ at $\sim 1 \mathrm{kHz}$, a dielectric strength of $13.4 \mathrm{~V} / \mu \mathrm{m}$. Because of the increased permittivity and high strain at break, these materials might find application as dielectric in energy harvesting devices operated at low electric fields. Furthermore, since the Ag core can in principle be replaced by other metals, the described procedure can be used for the synthesis of other polymer/metal composites and suggests a broad applicability of the method that would allow the synthesis of other functional materials.

\section{Acknowledgements}

We gratefully acknowledge D. Schreier and Dr. Y. Arroyo for helping with the TEM and SEM measurements, S. Dünki for the DMA measurements, B. Fischer for the TGA measurements, A. Wichser for helping with the ICP-OES 
measurements (all Empa) and M. Schneider from the Microlaboratory of the Laboratory for Organic Chemistry (ETH Zurich) for elemental analysis measurements. We thank B. Sinnet from Eawag for helping with DLS measurement. DMO thanks Dr. Racles from PPI for her kind support with the Sciex project. We also gratefully acknowledge Swiss National Science Foundation (SNF132101), Sciex (12.192), and Swiss Federal Laboratories for Materials Science and Technology (Empa, Dübendorf) for financial support.

\section{Notes and references}

${ }^{a}$ Swiss Federal Laboratories for Materials Science and Technology (Empa), Laboratory for Functional Polymers, Überlandstrasse 129, 8600 Dübendorf, Switzerland

${ }^{b}$ Ecole Polytechnique Fédérale de Lausanne (EPFL), Materials Institute, 1015 Lausanne, Switzerland

${ }^{c}$ Swiss Federal Laboratories for Materials Science and Technology (Empa), Laboratory for Advanced Analytical Technologies, Überlandstrasse 129, 8600 Dübendorf, Switzerland

Electronic Supplementary Information (ESI) available: Elemental analysis, TEM micrographs, EDX measurements, XPS spectra and further mechanical tensile tests. See DOI: 10.1039/b000000x/

1 S. H. Chae, W. J. Yu, J. J. Bae, D. L. Duong, D. Perello, H. Y. Jeong, Q. H. Ta, T. H. Ly, Q. A. Vu, M. Yun, X. Duan and Y. H. Lee, Nat. Mater., 2013, 12, 403-9.

2 P. Brochu and Q. Pei, Macromol. Rapid Commun., 2010, 31, 10-36.

3 J. Biggs, K. Danielmeier, J. Hitzbleck, J. Krause, T. Kridl, S. Nowak, E. Orselli, X. Quan, D. Schapeler, W. Sutherland and J. Wagner, Angew. Chemie - Int. Ed., 2013, 52, 9409-9421.

4 F. Carpi, D. De Rossi, R. Kornbluh, R. Pelrine and P. Sommer-Larsen, Eds., Dielectric Elastomers as Electromechanical Transducers, Elsevier, Amsterdam, 2008.

5 R. Pelrine, R. D. Kornbluh, J. Eckerle, P. Jeuck, S. Oh, Q. Pei and S. Stanford, SPIE's 8th Annu. Int. Symp. Smart Struct. Mater., 2001, 4329, $148-156$

6 M. Molberg, D. Crespy, P. Rupper, F. Nüesch, J.-A. E. Månson, C. Löwe and D. M. Opris, Adv. Funct. Mater., 2010, 20, 3280-3291.

7 H. Stoyanov, P. Brochu, X. Niu, E. Della Gaspera and Q. Pei, Appl. Phys. Lett., 2012, 100, 2010-2013.

8 K. Goswami, A. L. Skov and A. E. Daugaard, Chem. - A Eur. J., 2014, 20, 9230-9233.

9 A. G. Bejenariu, L. Yu and A. L. Skov, Soft Matter, 2012, 8, 3917.

10 F. Galantini, F. Carpi and G. Gallone, Smart Mater. Struct., 2013, 22, 104020.

11 F. B. Madsen, L. Yu, A. E. Daugaard, S. Hvilsted and A. L. Skov, RSC Adv., 2015, 5, 10254-10259.

12 B. Kussmaul, S. Risse, G. Kofod, R. Waché, M. Wegener, D. N. McCarthy, H. Krüger and R. Gerhard, Adv. Funct. Mater., 2011, 21, $4589-4594$.

13 F. B. Madsen, I. Dimitrov, A. E. Daugaard, S. Hvilsted and A. L. Skov, Polym. Chem., 2013, 4, 1700-1707.

14 C. Racles, M. Cazacu, B. Fischer and D. M. Opris, Smart Mater. Struct., 2013, 22, 104004.

15 G. Gallone, F. Carpi, D. De Rossi, G. Levita and A. Marchetti, Mater. Sci. Eng. C, 2007, 27, 110-116.

16 F. Carpi, G. Gallone, F. Galantini and D. De Rossi, Adv. Funct. Mater., 2008, 18, 235-241.

17 W. Hu, S. N. Zhang, X. Niu, C. Liu and Q. Pei, J. Mater. Chem. C, 2014, 2, 1658-1666.
18 F. Galantini, S. Bianchi, V. Castelvetro, I. Anguillesi and G. Gallone, Adv. Sci. Technol., 2012, 79, 41-46.

19 K. Goswami, A. E. Daugaard and A. L. Skov, RSC Adv., 2015, 5, 12792-12799.

20 D. M. Grannan, J. C. Garland and D. B. Tanner, Phys. Rev. Lett., 1981, 46, 375-379.

21 Z.-M. Dang, L. Wang, Y. Yin, Q. Zhang and Q.-Q. Lei, Adv. Mater., 2007, 19, 852-857.

22 X. H. Yang, H. T. Fu, K. Wong, X. C. Jiang and A. B. Yu, Nanotechnology, 2013, 24, 415601.

23 K. Berdel, S. S. Member, J. G. J. Rivas, P. H. Bolívar, P. De Maagt, S. S. Member and H. Kurz, IEEE Trans., 2005, 53, 1266-1271.

24 J. K. Nelson, R. J. Linhardt, L. S. Schadler and H. Hillborg, IEEE Trans. Dielectr. Electr. Insul., 2012, 19, 960-967.

25 L. J. Romasanta, M. Hernández, M. A. López-Manchado and R. Verdejo, Nanoscale Res. Lett., 2011, 6, 508.

26 Y. Zhou, Y. Chen, H. Wang and C. P. Wong, Mater. Lett., 2014, 119, 64-67.

27 D. M. Opris, D. Crespy, C. Löwe, M. Molberg and F. Nüesch, Proc. SPIE, Electroact. Polym. Actuators Devices (EAPAD)Electroactive Polym. Actuators Devices, 2009, 7287, 72870L-72870L-8.

28 C. Huang, Q. M. Zhang and J. Su, Appl. Phys. Lett., 2003, 82, 3502 3504 .

29 T. Kempa, D. Carnahan, M. Olek, M. Correa, M. Giersig, M. Cross, G. Benham, M. Sennett, Z. Ren and K. Kempa, J. Appl. Phys., 2005, 98, $3-$ 6.

30 J. Y. Li, L. Zhang and S. Ducharme, Appl. Phys. Lett., 2007, 90, 28-31.

31 L. Qi, B. I. Lee, S. Chen, W. D. Samuels and G. J. Exarhos, Adv. Mater., 2005, 17, 1777-1781.

32 N. Guo, S. A. DiBenedetto, P. Tewari, M. T. Lanagan, M. A. Ratner and T. J. Marks, Chem. Mater., 2010, 22, 1567-1578.

33 R. B. Grubbs, J. Polym. Sci. Part A Polym. Chem., 2005, 43, 4323-4336.

34 I. Pastoriza-Santos, J. Pérez-Juste, G. Kickelbick and L. M. Liz-Marzán, J. Nanosci. Nanotechnol., 2006, 6, 453-458.

35 J. Lu, K.-S. Moon and C. P. Wong, J. Mater. Chem., 2008, 18, 48214826.

36 J. Ellison, G. Wykoff, A. Paul, R. Mohseni and A. Vasiliev, Colloids Surfaces A Physicochem. Eng. Asp., 2014, 447, 67-70.

37 C. E. Hoppe, C. Rodríguez-Abreu, M. Lazzari, M. A. López-Quintela and C. Solans, Phys. Status Solidi, 2008, 205, 1455-1459.

38 B. Wiley, Y. Sun, B. Mayers and Y. Xia, Chem. - A Eur. J., 2005, 11, 454-63.

39 D. Kim, S. Jeong and J. Moon, Nanotechnology, 2006, 17, 4019-24.

40 Y. Shen, Y. Lin, M. Li and C.-W. Nan, Adv. Mater., 2007, 19, 1418 1422.

41 H. Baida, P. Billaud, S. Marhaba, D. Christofilos, E. Cottancin, A. Crut, J. Lermé, P. Maioli, M. Pellarin, M. Broyer, N. Del Fatti, F. Vallée, A. Sánchez-Iglesias, I. Pastoriza-Santos and L. M. Liz-Marzán, Nano Lett., 2009, 9, 3463-9.

42 A. Guerrero-Martínez, J. Pérez-Juste and L. M. Liz-Marzán, Adv. Mater., 2010, 22, 1182-95.

43 M. Cazacu, C. Racles, A. Vlad, M. Antohe and N. Forna, J. Compos. Mater., 2009, 43, 2045-2055.

44 J. Xu and C. P. Wong, Appl. Phys. Lett., 2005, 87, 082907.

45 C. Putson, D. Jaaoh, N. Meauma and N. Muensit, J. Inorg. Organomet. Polym. Mater., 2012, 22, 1300-1307.

46 Y. Zhang, Y. Wang, Y. Deng, M. Li and J. Bai, ACS Appl. Mater. Interfaces, 2012, 4, 65-68.

47 M. A. Lucchini, A. Testino, C. Ludwig, A. Kambolis, M. El-Kazzi, A. Cervellino, P. Riani and F. Canepa, Appl. Catal. B Environ., 2014, 156157, 404-415.

48 B. K. Park, S. Jeong, D. Kim, J. Moon, S. Lim and J. S. Kim, J. Colloid Interface Sci., 2007, 311, 417-24.

49 C. Liu, X. Yang, H. Yuan, Z. Zhou and D. Xiao, Sensors, 2007, 7, 708718

50 J. E. Q. Quinsaat, F. A. Nüesch, H. Hofmann and D. M. Opris, RSC $A d v ., 2013,3,6964-6971$.

51 C. Graf, D. L. J. Vossen, A. Imhof and A. van Blaaderen, Langmuir, 2003, 19, 6693-6700.

52 S. Liu and M.-Y. Han, Chem. Asian J., 2010, 5, 36-45.

53 V. Gun'ko, M. Vedamuthu, G. Henderson and J. Blitz, J. Colloid Interface Sci., 2000, 228, 157-170. 
54 J. Cervantes, R. Zárraga and C. Salazar-Hernández, Appl. Organomet. Chem., 2012, 26, 157-163.

55 M. C. Boyce, O. Yeh, S. Socrate, K. Kear and K. Shaw, J. Mech. Phys. Solids, 2001, 49, 1343-1360.

56 N. . Grassie and I. G. . Macfarlane, Eur. Polym. J., 1978, 14, 875-884.

57 J. P. Lewicki, J. J. Liggat and M. Patel, Polym. Degrad. Stab., 2009, 94, $1548-1557$.

58 V. P. Silva, M. C. Gonçalves and I. V. P. Yoshida, J. Appl. Polym. Sci., 2006, 101, 290-299.

59 M. Norkhairunnisa, A. Azizan, M. Mariatti, H. Ismail and L. Sim, J. Compos. Mater., 2011, 46, 903-910.

60 C. Calebrese, L. Hui, L. Schadler and J. Nelson, IEEE Trans. Dielectr. Electr. Insul., 2011, 18, 938-945.

61 Q. Tan, Y. Cao and P. Irwin, 2007 IEEE Int. Conf. Solid Dielectr., 2007, $411-414$.

62 M. Kurimoto, H. Okubo, K. Kato, M. Hanai, Y. Hoshina, M. Takei and N. Hayakawa, IEEE Trans. Dielectr. Electr. Insul., 2010, 17, 662-670.

63 M. Kollosche and G. Kofod, Appl. Phys. Lett., 2010, 96, 12-15.

64 J. Claude, Y. Lu and Q. Wang, Appl. Phys. Lett., 2007, 91, 2-5.

65 K. H. . Stark and C. G. Garton, Nature, 1955, 175, 642-643.

66 R. Kornbluh, R. Pelrine, Q. Pei, R. Heydt, S. Stanford, S. Oh and J. Eckerle, Proceeding SPIE, Smart Struct. Mater., 2002, 4698, 254-270.

67 R. Pelrine, R. D. Kornbluh, J. Eckerle, P. Jeuck, S. Oh, Q. Pei and S. Stanford, SPIE's 8th Annu. Int. Symp. Smart Struct. Mater., 2001, 4329, $148-156$. 